\title{
Medición del servicio de internet durante un mes de clases presenciales asistidas a través de tecnología remota
}

\section{Internet service measurement for a month of face-to-face assisted classes through remote technology}

\author{
DOI: http://doi.org/10.17981/bilo.2.1.2020.2
}

Artículo de Investigación Científica. Fecha de Recepción: 01/05/2020. Fecha de Aceptación: 15/05/2020

Antony Lombana-Jiménez

Universidad de la Costa CUC. Barranquilla, (Colombia)

alombana@cuc.edu.co

Levith Sarmiento-Escobar

Universidad de la Costa CUC. Barranquilla, (Colombia)

lsarmien@cuc.edu.co

Carlos Castro-Torres

ccastro37@cuc.edu.co

Universidad de la Costa CUC. Barranquilla, (Colombia)

Alexander Troncoso-Palacio

Universidad de la Costa CUC. Barranquilla, (Colombia)

atroncos1@cuc.edu.co

Para citar este artículo:

A. Lombana-Jiménez, L. Sarmiento-Escobar y A. Troncoso-Palacio, "Medición del servicio de internet durante un mes de clases presenciales asistidas a través de tecnología remota", INGE CUC, vol. 2, no. 1, 2020. DOI: http://doi.org/10.17981/bilo.2.1.2020.1

\section{Resumen}

Con el creciente aumento de la tecnología, la industria de la comunicación a través de internet se hace cada vez más necesaria utilizarla en la familia, el trabajo incluso en los estudios, de tal manera que es indispensable mantener una conexión estable. Pero debido al confinamiento que se vive a principios del año 2020, por causa de la Pandemia de Coronavirus declarada por la Organización Mundial de la Salud, lo cual, ha ocasionado un aumento en la cantidad de usuarios conectados, ocasionando inestabilidad en el servicio. Por ello, se realizó este estudio iniciando con una encuesta a 180 usuarios con servicio contratado de 5 megas. Con la ayuda de herramientas de control de calidad, como los gráficos de control X S, se evidenció que de 9 am hasta las 6 pm, son las horas donde se generaba mayor fluctuación de la conexión, pues el servicio presentó inestabilidad y desciende alrededor de los 3.5 megas. Al aplicar el Diagrama de Ishikawa se encontraron una serie de causas que generaban dicho problema. Posteriormente, se realizó un graficó mediante un Diagrama de Pareto, donde se evidenció que 
BILO Vol. 2 No. 1. Enero- Junio de 2020

aproximadamente el $80 \%$ de los problemas son ocasionados por sobre uso del servicio, falta de mantenimiento y materiales de mala calidad

Palabras clave: Conectividad a internet, herramientas de calidad, causas-efecto

\begin{abstract}
The communication industry has changed completely with the arrival of the internet. Online communication is in demand nowadays becoming a necessity in people's daily activities and lives at their homes, work places, and schools. These are the markets that make online communication an essential tool to the community. As a result of the lockdown; due to the sanitary emergency that has caused COVID-19, the internet connection has shown irregularities. In this article; with the help of quality control tools such as the XS control chart, we were able to establish the exact times where the connection was most fluctuated. In addition, using the Ishikawa diagrams we found the potential causes that were creating these irregularities. Also, using a Pareto diagram led us to find the different shortcomings of the company providing the internet connection services. Based on the quality control tools implemented in this research, recommendations have been made for the improvement of this process; such as having a better planning that includes employee training, maintenance plans of the equipment, and verification of the capacity and quality of those equipment.
\end{abstract}

Keywords: Internet connection, Quality tools, Causes-Effect Diagram

\title{
I. INTRODUCCIÓN
}

El internet ha sido una herramienta que ha revolucionado el mundo en general, lo podemos ver con el sistema de comunicación por medio de las distintas plataformas digitales las cuales han sido fundamentales sobre todo para las industrias y que muchas de estas se ven afectadas cuando este no se encuentra presente, debido a esto se busca constantemente que la conexión a internet no presente intermitencia para que los procesos en las industrias no se vean afectados, sin embargo ha sido complicado controlar esto ya que no se sabe con certeza cuál es la causa principal de dichas fallas, el ejemplo más claro en estos momentos se ha presentado con la emergencia sanitaria declarada a nivel mundial por la OMS (organización mundial de la salud) por la pandemia COVID-19, donde nos hemos visto en la necesidad de permanecer en casa sin dejar de lado las actividades diarias entre las que tenemos trabajos, estudios, entre otros y se ha hecho necesario la utilización del internet para permanecer en constante comunicación, lo cual ha sido un completo caos, para esto es necesario la utilización de herramientas de calidad como lo son el Ishikawa y diagrama de Pareto, donde por medio de estas herramientas se nos permite hacer una investigación de esas causas que dependiendo de su proceder se pueden denominar normales o especiales, y así enfocarnos en repelar a las causas que más lo ameriten.

\section{ESTUDIOS PRIMARIOS DE LA LITERATURA}

Se define calidad como la capacidad de satisfacer a un cliente con un producto o servicio que cumpla a cabalidad las particularidades y características necesarias con el fin suplir todo tipo de necesidad necesidades [1]. Por otro lado, el control es la evaluación de procedimientos o procesos, el cual se llevan a cabo con el fin de detectar desvíos con respecto a las especificaciones de un plan de acción establecido, desvíos que serán corregidos mediante la aplicación de herramientas de control [2]. Por lo tanto, se puede definir como control de calidad el conjunto de actividades y mecanismos que se llevan a cabo aplicando herramientas de control con el fin de detectar e identificar errores, y lograr el cumplimiento total de las características de un producto o servicio que logre la satisfacción de un cliente, las empresas con el fin de mantenerse competitivas tranzan su ruta estratégica basándose en la calidad de sus productos y prestación de servicios por tal motivo hace parte de su planeación el seguimiento constantes del comportamiento de sus procesos con el propósito de mantenerse en la mejora continua [3]. en el presente artículo analizaremos a través de herramientas de control de calidad el problema que presenta la empresa Cable Éxito en barranquilla que pertenece a la industria telefónica, dicha empresa presta el servicio de conectividad a internet; servicio el cual presenta variación en horas del día afectando de esta manera la conectividad a internet de sus usuarios que a causa de la emergencia sanitaria declarada por la propagación del COVID-19 han tenido que recurrir al 


\section{BILO Vol. 2 No. 1. Enero- Junio de 2020}

trabajo remoto, haciendo de la conectividad a internet un servicio de primera necesidad. Inicialmente utilizamos una gráfica de control de variables las cuales se utilizan para visualizar variaciones con respecto a una tendencia central [4] teniendo en cuenta observaciones realizadas por medio de un conjunto de datos recolectados [5] para identificar en ciertas horas del día como era la variación de megabyte con el fin de identificar que tanta intermitencia existe en la velocidad del internet, la gráfica de control utilizada fue $(\bar{X})$ o promedio y $s$ (desviación estándar) que se utilizan para observar la variación del promedio de dichas muestras con el objetivo de obtener una explicación a tal variación [6]. luego de obtener esta información, se hizo necesario la utilización de la herramienta Ishikawa, también conocido como diagrama de espina de pescado por su forma característica que posee. Creado por el Dr. Kaoru Ishikawa, para representar gráficamente de una forma clara y precisa los factores que afectan en la prestación del servicio de conexión a internet [7], Para la construcción del diagrama de Ishikawa debemos colocar en la cabeza el problema o efecto al que queremos buscarle las causas, definir cada una de las flechas (6M's) que interviene en el proceso y así mismo su subcausas, cada una organizada en su respectiva categoría [8]; por ultimo para la tomas de ciciones y desarrollo de un nuevo plan de acción con el fin de solucionar el efecto que presentan las causas mostradas por la herramienta diagrama de Ishikawa, es necesario la aplicación del diagrama de Pareto o el diagrama 80-20, el cual es una gráfica que permite ver las frecuencias distribuidas de los datos recolectados según la problemática investigada junto con sus causas [9], esta grafica mostrara las frecuencias de manera descendente, en donde las primeras causas o defectos sumaran entre el 70 y 80 por ciento de todas las causas existentes y que de acuerdo con su concepto es más útil para llegar a identificar cual o cuales son las causas principales en el problema [10] investigado y así poder llegar a la planeación de actividades con el propósito de mitigar esas causas potenciales que afectan la prestación del servicio y lograr la mejora del mismo [11]-[12].

\section{METODOLOGIA}

De acuerdo con la problemática encontrada se hizo la eleccion de una de las empresas prestadora del servicio de conexión a internet, a la cual se le realizo una investigacion para obtener una descripcion de su actividad operativa a la cual se le va a realizar el analisis, utilizando las herramientas de calidad para la obtencion y organización de informacion que nos permitiera dar por medio de estas con las diferentes causas que afectan para que el proceso no se lleve a cabalidad, mostrando asi la efectividad que estas tienen en el analisis de informacion. Lo cual se evidencia en el siguiente diagrama:

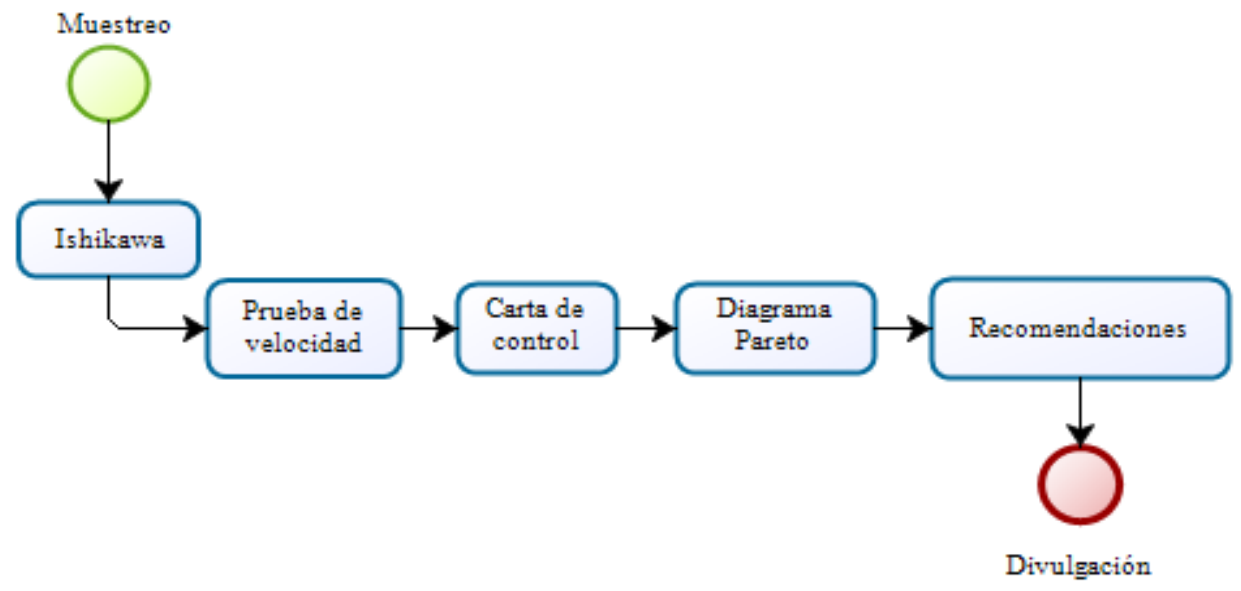

Fig. 1 Flujograma de metodología, Elaborado por: Autores

\section{DESARROLLO}

Se realizó una toma de medidas de la velocidad de internet a cada hora de 8 am hasta 9 pm durante cuatro semanas de lunes a viernes, teniendo en cuenta que el analisis se hará al servicio ofrecido de 5 megas. Se procede a determinar los limites de acuerdo con la formulas que se utilizan para graficar las cartas de control X S, con la cual se obtuvieron los siguientes datos: 
BILO Vol. 2 No. 1. Enero- Junio de 2020

\begin{tabular}{|c|c|c|c|c|c|}
\hline$\mu$ & LSX & LIX & S & LSS & LIS \\
\hline 5 & 4,65335714 & 3,50378571 & 0,92857143 & 1,34178571 & 0,51535714 \\
\hline
\end{tabular}

Despues de haber realizado la toma de estas medidas, se obtuvieron las diferentes graficas de control X S.

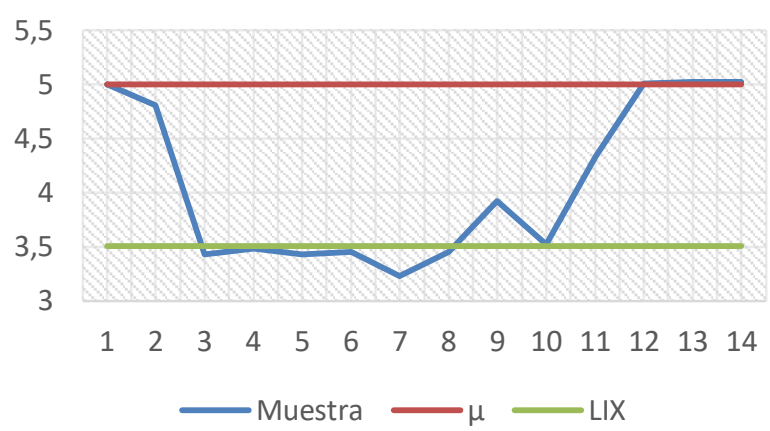

Figura 1. Datos con respecto al objetivo

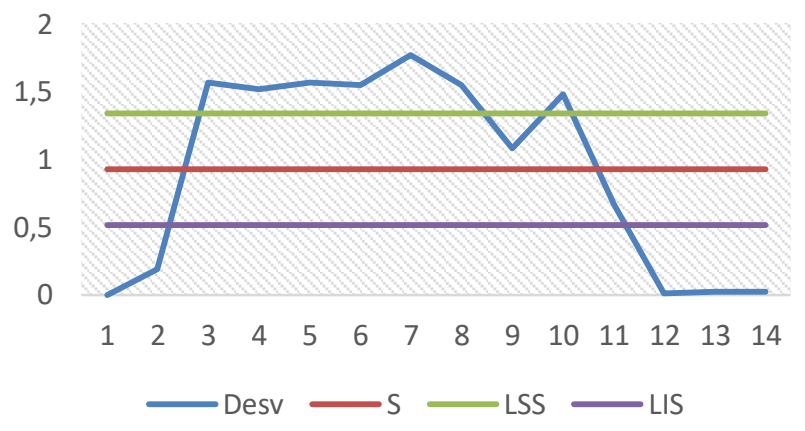

Figura 2. Variabilidad de las mediciones

Analizando estas graficas obtenemos que la mayor fluctuacion de la conexcion del internet se presenta desde las 9am y $10 \mathrm{pm}$, indicando de esta manera que es cuando el servicio tiene una mayor utlizacion por parte de los usuarios y es cuando mayor variabilidad se presenta durante el servicio y cae al redor de los 3.5 megas ocacionando lentitud e inestabilidad en la conexión a internet, razon por la cual se procede a realizar un diagrama causa efecto, que se evidencia en la figura 3

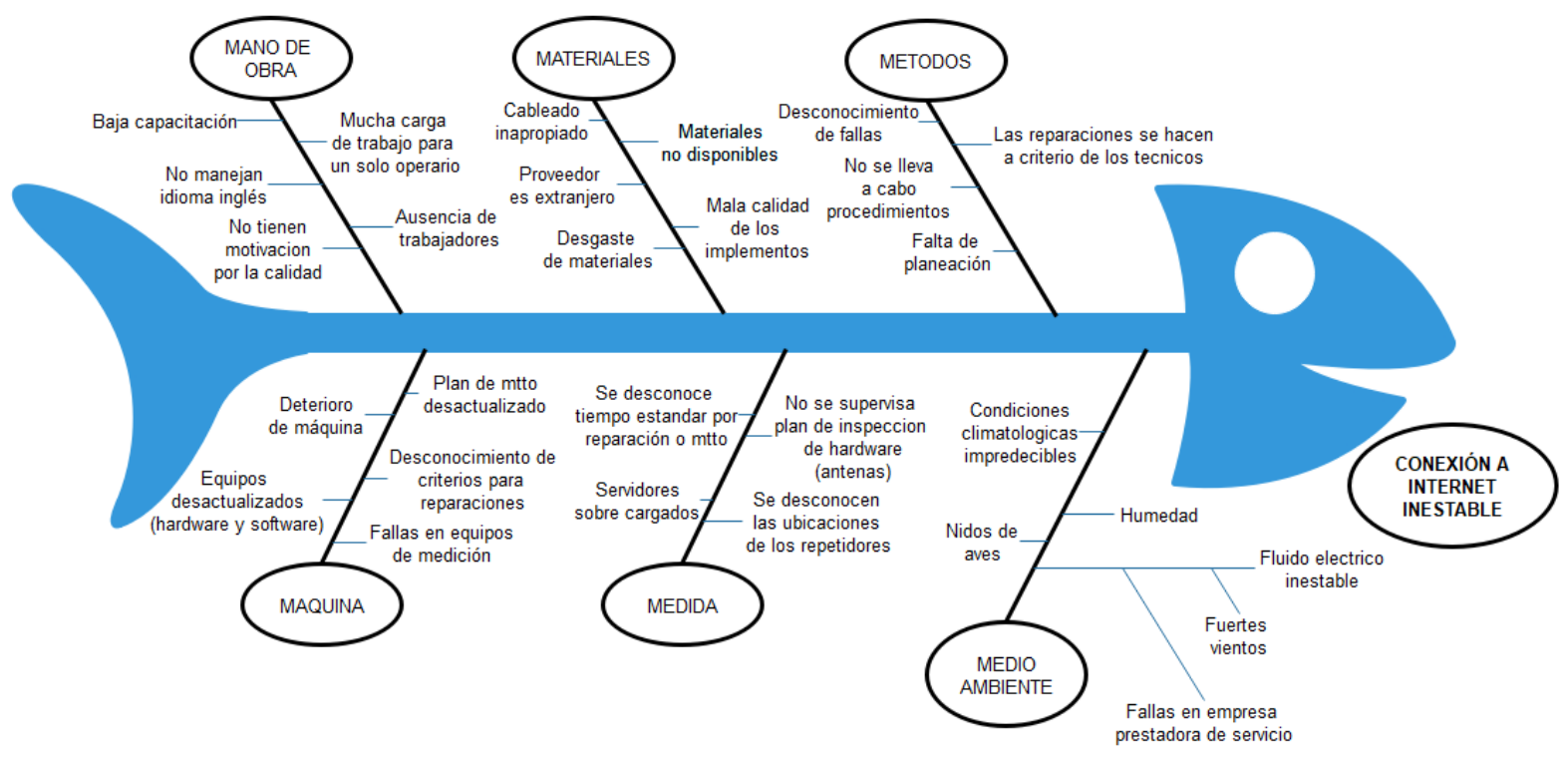

Figura. 3 Diagrama de Ishikawa Elaboración: Autores

Con el diagrama de ishikawa se obtuvo la informacion, clasificando las causas principales y sus respectivas subcausas que son las generadoras de la conexión inestable a internet. El paso siguiente es hacer la gráfica de Pareto dónde se ordenaron las causas por su intensidad de frecuencia y se evidencian en la figura 4. 
BILO Vol. 2 No. 1. Enero- Junio de 2020

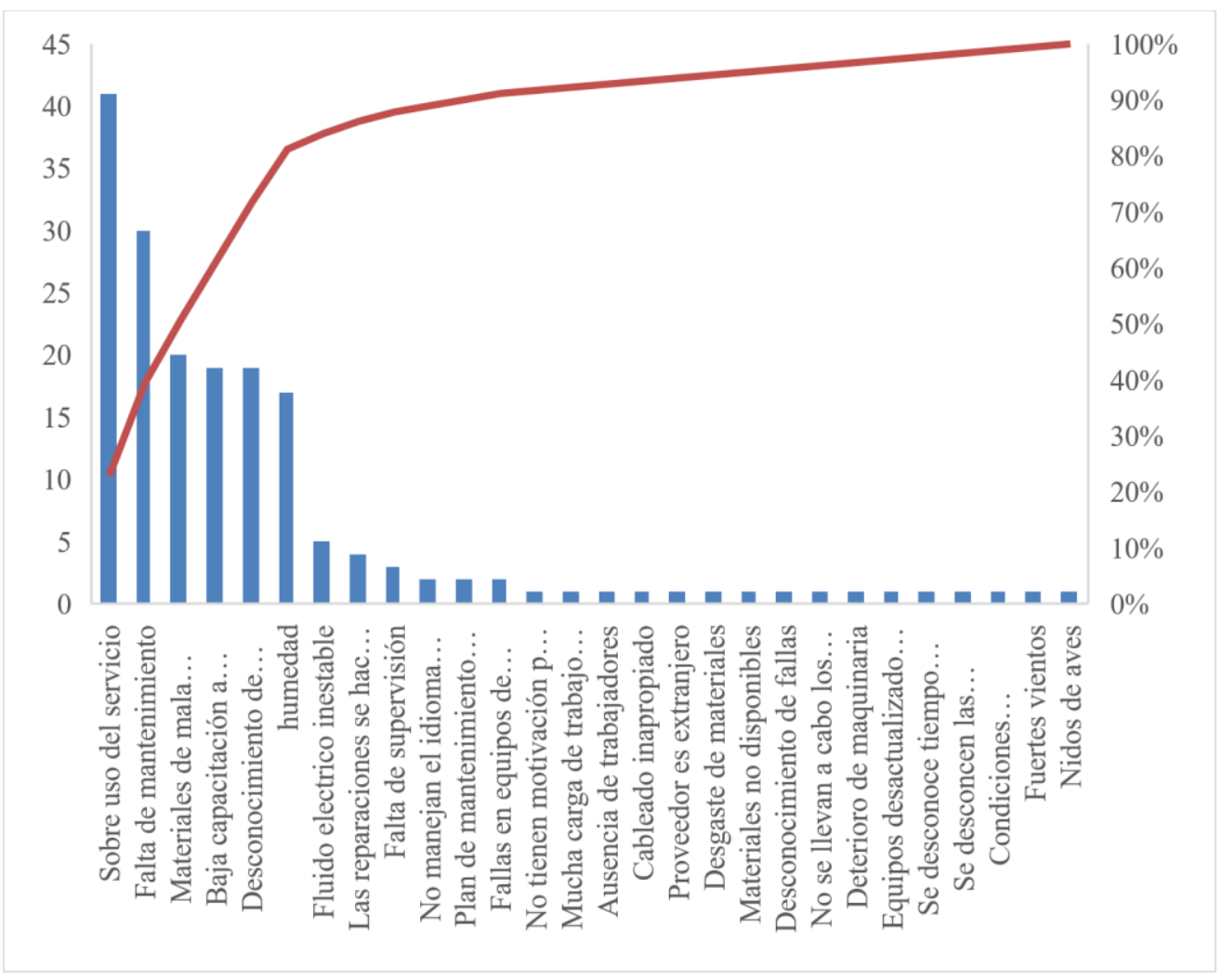

Figura 4. Identificación de causas a través del diagrama de Pareto. Elaborado por: Autores

A través de esta herramienta se logró identificar las causas potenciales que afectan el servicio de conectividad de los usuarios, notando que de veintiséis (26) causas identificadas, seis (6) de ellas son vitales y se deben tener en cuenta para la mejora del proceso. En la tabla 2 se identifican cada una de las causas posibles de la intermitencia de la conectividad a internet.

\begin{tabular}{lc}
\hline \multicolumn{1}{c}{ Causas según encuesta } & Frecuencia \\
\hline Sobre uso del servicio & 41 \\
Falta de mantenimiento & 30 \\
Materiales de mala calidad & 20 \\
Baja capacitación a usuarios & 19 \\
Desconocimiento de reparaciones & 19 \\
humedad & 17 \\
Fluido eléctrico inestable & 5 \\
Las reparaciones se hacen a criterio de los trabajadores & 4 \\
Falta de supervisión & 3 \\
No manejan el idioma inglés & 2 \\
Plan de mantenimiento desactualizado & 2 \\
Fallas en equipos de medición & 2 \\
No tienen motivación por la calidad & 1 \\
Mucha carga de trabajo para un solo operario & 1 \\
Ausencia de trabajadores & 1 \\
Cableado inapropiado & 1 \\
Proveedor es extranjero & 1 \\
Desgaste de materiales & 1
\end{tabular}


BILO Vol. 2 No. 1. Enero- Junio de 2020

\begin{tabular}{lc} 
Materiales no disponibles & 1 \\
Desconocimiento de fallas & 1 \\
No se llevan a cabo los procedimientos establecidos & 1 \\
Deterioro de maquinaria & 1 \\
Equipos desactualizados (hardware y software) & 1 \\
Se desconoce tiempo estándar por reparación o mantenimiento & 1 \\
Se desconocen las ubicaciones de los repetidores & 1 \\
Condiciones climatológicas impredecibles & 1 \\
Fuertes vientos & 1 \\
Nidos de aves & 1 \\
\multicolumn{1}{c}{ Total } & 180 \\
\hline
\end{tabular}

Tabla 2. Nombre de las distintas causas identificadas

\section{CONCLUSIONES}

Después de haber obtenidos los resultados mediante la utilización de las distintas herramientas de cálida como los gráficos X S, diagrama de Ishikawa y el diagrama de Pareto, lo cual son completamente indispensables si queremos que nuestro proceso, actividad, servicio entre otros se encuentren de manera controlada, entendiendo que estas herramientas no se utilizan solo para recoger datos, sino que esos datos se conviertan en información que sirvan para la mejora continua de lo que se desee mantener en control. Por lo tanto, con base en los resultados obtenidos se les recomienda a las empresas que prestan el servicio de conexión de 5 megas a internet en los barrios de estrato 2 de la ciudad de Barranquilla, no sobre pasar la capacidad de usuarios para que no se presente sobrecarga a los servidores, realizar planes de mantenimiento a las redes y verificar la capacidad y calidad de los equipos utilizados en el proceso.

\section{REFERENCIAS}

[1] J. Evans y W. Lindsay, Administración y control de la calidad, 9 ed., Mexico: CENGAGE learning, 2015, p. 792.

[2] E. Jara, «El control en los procesos administrativos,» Academia, 15 Agosto 2009. [En línea]. Available: https://www.academia.edu/29995190/EL_CONTROL_EN_EL_PROCESO_ADMINISTRATIVO.

[3] S. Val y J. Valtueña, Calidad, 1 ed., Madrid: Mc Graw Hill, 2007, p. 224.

[4] H. Gutiérrez Pulido y R. De La Vara Salazar, Control estadístico de la calidad y Seis Sigma, 3 ed., Mexico: McGraw Hill, 2013, p. 488.

[5] D. H. Besterfield, Control De Calidad, 8 ed., Mexico: Pearson Educación, 2009, p. 552.

[6] L. E. Romero Vega, L. C. Valdés Luna, J. G. Pastor de Moya y R. J. Herrera Acosta, «Statistical control for monitoring the process of cutting soap bars,» Ingeniería y Desarrollo, vol. 36, $\mathrm{n}^{\circ} 2$, pp. 455-468, Julio-Diciembre 2018.

[7] P. Valderrey, Herramientas para la calidad total, 1 ed., Bogota: Ediciones de la U, 2013, p. 296.

[8] R. Maldonado y L. Graziani, «Herramientas estadísticas de la calidad para la diagnosis: estudio de un caso en la industria de productos cárnicos,» Interciencia, vol. 32, n 10, pp. 707-711, 10 Octubre 2007. 
BILO Vol. 2 No. 1. Enero- Junio de 2020

[9] K. Ishikawa, Introduccion al Control de Calidad, 1 ed., Madrid: Diaz de Santos, 1994, p. 500.

[10] H. Gutiérrez, Calidad y productividad, 4 ed., Mexico: Mc Graw Hill, 2014, p. 382.

[11] J. M. Izar y J. H. Gonzalez, Las Siete Herramientas Básicas de la Calidad, 1 ed., Mexico: Universidad Potosina, 2004, p. 216.

Jiménez Ariaz, G., Ortiz Severiche, W., Pérez Gómez, E., Romo Boneth, E., \& Crespo Arévalo, L. (2018).

[12] Pertinencia de las tecnologías de la información y la comunicación para el fortalecimiento de la comprensión lectora. IJMSOR: International Journal of Management Science \& Operation Research, 3(1), 57-63. Recuperado a partir de http://ijmsoridi.com/index.php/ijmsor/article/view/97

[13] Patiño Builes, A. (2015). Technology trends for business productivity increase. INGE CUC, 11(2), 84-96. https://doi.org/10.17981/ingecuc.11.2.2015.09 